\section{Unusual and severe peripartum cardiomyopathy: A case report}

\author{
Lamanna B, Vinciguerra M, Crupano F, Cicinelli R, Cicinelli E \\ and Vimercati $A$
}

Department of Biomedical Sciences and Human Oncology, Bari, Italy

\begin{abstract}
Peripartum cardiomyopathy (PPCM) is a relatively rare cardiac disease that manifests in the final stage of pregnancy and in the first months after delivery in women with no preexisting heart disease. Many etiological processes have been suggested: viral myocarditis, abnormal immune response to pregnancy, excessive prolactin excretion, prolonged tocolysis and a familiar predisposition to PPCM. Its diagnosis is often delayed because its symptoms, which include fatigue, dyspnea and palpitations are nonspecific. For this reason the diagnosis of PPCM is still made by exclusion of other etiologies. The long-term prognosis, once the acute phase is over, is a function of myocardial damage, this varies from complete functional recovery to chronic HF. The outcome of PPCM is highly variable with an alevated risk of fetomaternal morbidity and mortality. We report a serious case of a 40 years old female with biamniotic bicorionic twin pregnancy (PMA) who delivered by caesarean section and developed acute PPCM on post-operative. Symptoms occurred two hours after an intramuscular injection of two vials of methylergonovine the same day of cesarean delivery. These manifested in sudden tachypnoe, tachycardia and the appearance itchy maculopapular rash on her chest. On further evaluation, ECHO revealed cardiomegaly with reduced ejection fraction $(<15 \%)$. The case was successfully managed by a multidisciplinary team, using drugs like levosimendan and cabergoline, which rapresent emerging strategy in this clinical context.
\end{abstract}

More Information

*Address for Correspondence: Bruno Lamanna, Department of Biomedical Sciences and Human Oncology, Bari, Italy,

Email: lamannabruno@gmail.com

Submitted: March 11, 2021

Approved: May 04, 2021

Published: May 05, 2021

How to cite this article: Lamanna B, Vinciguerra M, Crupano F, Cicinelli R, Cicinelli E, et al. Unusual and severe peripartum cardiomyopathy: A case report. J Cardiol Cardiovasc Med. 2021; 6: 040-043.

DOI: 10.29328/journal.jccm.1001116

Copyright: @ 2021 Lamanna B, et al. This is an open access article distributed under the Creative Commons Attribution License, which permits unrestricted use, distribution, and reproduction in any medium, provided the original work is properly cited.

Check for updates

OPEN ACCESS

\section{Introduction}

Peripartum cardiomyopathy (PPCM) is an idiopathic cardiomyopathy conferred with heart failure secondary to left ventricular (LV) systolic dysfunction near the end of a pregnancy or during the months following delivery. Many etiological processes have been suggested: viral myocarditis, abnormal immune response to pregnancy, excessive prolactin excretion [1] and prolonged tocolysis. A familiar predisposition to PPCM has also been reported [2]. The disorder carries a high mortality rate [3-5]. Its diagnosis is made by exclusion of any other cause of heart failure combined with the LVEF almost reduced to below $45 \%$ but not consistently associated with LV dilatation. The estimated incidence of PPCM shows strong differences on a territorial basis: in Europe and in the US it is associated with one in every 3000 to 4000 live births. The distinctly higher incidence rates in Haitian and South African women (up to 1: 300) suggest that genetic or environmental factors may play a role in the pathogenesis of the disease. However, the etiology of the PPCM is still obscure $[6,7]$.

The definition of PPCM includes four criteria $[8,9]$ :

1. development of cardiac failure in the last month of pregnancy or within five months of delivery;
2. absence of an identifiable cause for the cardiac failure;

3. absence of a recognizable heart disease before the last month of pregnancy;

4. left ventricular (LV) dysfunction (ejection fraction of less than $45 \%$ or reduced shortening fraction);

Risk factors include multiple gestation, pre-eclampsia, obesity, advanced maternal age, African descent, prolonged tocolysis and pregnancy comordities such as pulmonary embolia $[10,11]$. The prognosis is related to the recovery of ventricular function. The mortality rate of peripartum cardiomyopathy is $30 \%-60 \%[12,13]$. Survivors have a $50 \%-80 \%$ risk of developing cardiac failure during future pregnancies, with an associated mortality rate of $60 \%[14,15]$.

The manifestation of peripartum cardiomyopathy is also highly variable [16]. In some women the clinical and echocardiographic status improves and sometimes returns to normal, whereas in others, the disease progresses to severe cardiac failure and even sudden death. In the present report, we describe a case of PPCM during the 35th week of gestation of twin pregnancies obtained after in vitro fertilization. 


\section{Presentation of case}

The report is about the case of a white woman, aged 40 with biamniotic bicorionic twin pregnancy achieved with FIVET and with a 4-cm cervical myoma. Her medical history was unremarkable. She presented signs of Klumpe's palsy (a type of obstetric brachial plexus palsy). She did not have a history of hypertension, congenital heart diseas, myocarditis, valvular heart disease, cardiomyopathy or autoimmune disease. She never had an abortion. She had no known allergies, did not smoke, drink alcohol, or use illicit drugs. Her familial anamnesis was negative for cardiovascular disease or for any cardiovascular risk factors. During the gestation period, the patient was asymtomatic, until the described episode. The first hospital admission was at 27 weeks because of referred uterine contractions. She received atosiban, a tocolytic agent, and antenatal corticosteroids for accelerating fetal lung maturation. On this occasion the routine Electrocardiogram (ECG) revealed a sinus rhythm of 110 beats per minute.

At $35 \mathrm{w}$ of amenorrea the patient was subsequentally admitted to hospital because one of the twins was in a transverse situation.

She underwent elective cesarean section at 12 am of 23/06/2018. Spinal anaesthesia was administered with a 25-gauge needle at L3-L4 interspace with $12 \mathrm{mg}$ of $0.5 \%$ of hyperbaric bupivacaine. The patient returned to ward with $20 \mathrm{UI}$ of syntocinon in $500 \mathrm{ml}$ of $0.9 \%$ sodium chloride solution. At 4 p.m. of the same day of cesarean delivery, the nurse gave that patient an intramuscular injection of two vials of methylergonovine $(1 \mathrm{ml})$ in the gluteus maximus. At 5:30 pm there was a sudden and unexpected impairment of the patient's clinical status. The patient was anxious and did not manage to speak and to explain what she felt because of severe dyspnea and tachypnea. A physical examination revealed an itchy maculopapular chest rash had rapidly developed, she presented no extremities edema, tenderness, cough or chest pain and no fever. The cardiac auscultation showed a fast rate, an irregular rythm and an S3 gallop. An EKG showed extrasystolic arhythmia. Moreover a chest auscultation presented bilateral basal fine crepitations and vesicular murmur was diffusely reduced in both lungs. Patient's blood pressure (BP) increased from 126/74 mmHg (before C-section) to $170 / 110 \mathrm{mmHg}$. Her pulse was 117 beats per minute, respiratory rate was 32 breaths per minute, hypoxemia and hypocapnia with a compensatory reduction of bicarbonates was documented by arterial blood gas analysis (ABG: PH 7,42, pCO2 $24 \mathrm{mmHg}, \mathrm{pO} 250 \mathrm{mmHg}, \mathrm{Cl} 108$ $\mathrm{mmol} / \mathrm{L}$, Lac 2,5 mmol/L, HCO3- 15,6 mmol/L). According to the anesthetist she was given supplemental oxygen via nasal cannula, furosemide ( 2 vials of $20 \mathrm{mg}$ ) and corticosteroid (1 vial of flebocortid $100 \mathrm{mg}$ ) intravenously. This greatly relieved the symptoms: the skin rash suddenly disappeared; a second EKG showed a sinus tachycardia (the initial arythmia converted spontaneously to sinus rhytm within 1 hour).
Computer tomography (CT) chest scan was negative for pulmonary embolia and described pulmonary congestion. The transthoracic echocardiography at that time showed a LV dilatation, global LV hypokinesis, and an estimated ejection fraction of $15 \%$. At 8 pm Plasma TroponinI was $1.9 \mathrm{ng} / \mathrm{L}$. The woman was shifed to the intensive care unit, where she started therapy with levosimendan, a calcium-sensitizing drug with inotropic properties, in order to improve the marked systolic disfunction. At 11 pm EKG revealed sinus rhythm of 93 beats per minute and another ABG showed (PO2 64, PCO2 23, $\mathrm{Ph} 7,45$, Lat 2,7). She also started ventilatory support in the form of bilevel positive airway pressure (BPAP FIO2 $50 \%$ IPAP 7 EPAP 5). She was managed conservatively with intravenous furosemide, corticosteroid, nebulization with salbutamol and oxygenation. A chest X-ray PA view was done which showed cardiomegaly with large pulmonary consolidation in the inferior right lobe with airbronchograms. At 6:30 am of 24/06/2018 circulating levels of B-type natriuretic peptide (BNP) were $1216 \mathrm{pg} / \mathrm{ml}$ and Plasma TroponinI was $3,6 \mathrm{ng} / \mathrm{mL}$. She was also given cabergoline in order to inhibit lactation and to facilitate ventricular recovery. The day after an ecocardiography showed a LVEF of $20 \%$ with slight mitral and tricuspid regurgitation, and her LVEF improved to 50\% 11 days postoperation. Cardiac MRI 15 days after discharge showed a LVEF 55\%.

Ventilatory support was continued for 48 hours and thereafter extubation was done. She was discharged on $12 / 07 / 2018$ consequent to her improved clinical status, her pulse rate was 55 beats per minute, respiratory rate was 18/ min and blood pressure 125/75 mmHg. Her chest had cleared and the cardiovascular system showed good hemodynamics.

At the time of discharge she was advised to continue furosemide tablet $25 \mathrm{mg}$ once daily, one tab bisoprolol 1,25 mg in the morning and two in the evening and ace-inhibitor (Ramipril) 2,5 mg once daily.

The follow-up carried out 6 months later showed she was asymtomatic, the echocardiogram at the same point showed an improved ejection of 55\%. She has interrumpted therapy with diuretics and ACE-inhibitor, but she continues to take beta-blocker at lower dose combined with AT1-receptor antagonist.

\section{Discussion}

PPCM is a rare condition that presents high risks, even leading to death. The onset of the disease can be subdued with nonspecific signs and symptoms that, if not subjected to careful evaluation, can be confused with "discomfort". Symptoms of PPCM include fatigue, dyspnea that are a common finding in normal pregnancy and even in the initial peripartum state, PPCM is often undiagnosed. When the clinical suspicion emerges, it is important to obtain a rapid clinical-laboratoryimaging diagnosis, in order to start the appropriate therapeutic support for the woman. This article describes the diagnostic 
process of a patient with PPCM and a successful management of a severe form of cardiac dysfunction seeking to put forward a number of ideas for consideration about unusual aspects like the skin rash at the presentation combined with typical heart failure signs and symptoms. In literature there aren't any PPCM cases strictly related to skin reaction, in our case the maculopapular chest rash could be explained as an idiosyncratic reaction of methilergonovine and this is supported both by the timing of rash onset coincident with the methilergonovine Tmax (the time after drug administration when the maximum plasma concentration is reached) and by the rash responsivness to corticosteroids treatment. Despite in literature there isn't a significant correlation between this drug and cardiovascular events, knowing pharmacodynamics properties of this active principle we can easily suppose that it could have uncovered and triggered heart failure symptom, accelerating PPCM onset.

In terms of PPCM treatment, recommendation are based on the European Society of Cardiology guide for the management of cardiovascular diseases during pregnancy and peri-partum period. Usual heart failure treatments during pregnancy includes betablockers, vasodilators and diuretics, these last ones at the lowest dose possible in order to reduce placental perfusion impairment; after delivery this therapy can be optimized adding ACE-inhibitors or AT1 receptor antagonists or aldosterone receptor blockers. When there is a hemodynamic imbalance clinicians often employed classic inotropic agents like dobutamine $[17,18]$. Because of the poor outcome of these patients and the available scientific literature lacks from enough strong evidence regarding PPCM for our patient we chose to combine this gold standard therapy with two other drugs according to recent evidence. First of all, considering the sudden and severe patient left ventricle unloading, it was necessary to modifying the force and speed of contraction muscles, therefore we started an early treatment with levosimendan, an inodilatator agent choosen for its ability to enhance myocardial contractility without increasing oxygen requirement. Levosimendan is an inodilator, which increases myocardial contractility by enhancing cardiac sensitivity to calcium. Despite the improvement in ventricular function, levosimendan does not increase myocardial oxygen uptake significantly.1 Intracellular calcium is unaffected, thus limiting arrhythmic potential. The positive inotropic effect is independent of $\beta$ receptors and cAMP (cyclic adenosine monophosphate). Levosimendan also opens ATP-sensitive vascular potassium channels, causing vasodilation in veins, arteries and coronary arteries. This results in afterload reduction [19]. Actually there are few publications regarding evidence-based use of levosimendan in PPCM, but according to the great results reported it could soon become a new therapeutic strategy in this clinical context [20,21]. On the other hand there are new evidence in favor of use of indirect prolactin inhibitors, like cabergoline and bromocriptine, to enhance more rapidly cardiac pump recovery in PPCM. This latter pleiotropic effect would also synergize in order to improve the cardiac inotropism and the cardiac output too with the pharmacodynamic effect, which these dopamine agonists dircetly exerts on the myocardium [22,23]. Indeed, although the pathophysiology of PPCM remains unclear and the avaible theories only partially explain the mechanisms under this cardiovascular disease, recent researches focus on the role of increased prolactin levels in pregnancy and during lactation period. PRL would be responsible for significant hemodynamic changes: decreased angiotensin responsiveness and consequent reduced blood pressure, a concomitant increased blood volume and an increased level of EPO with a consequent increased haematocrit; all these conditions, according to Virchow Triad, could lead to microvascular insults and endothelium damage. A recent study on mice describes how the low level of STAT3 enhances expression of cardiac cathepsin D and consequently the cleavage of PRL 23kDa in PRL 16kDa relates to cardiac dysfunction and vascular damage [24,25]. Therefore, considering this PRL fragments involved in PPCM pathophysiology, we treated our patient with cabergoline, a strong and long lasting dopamine receptor agonist, which help her to accelerate cardiac recovery decreasing pituitary PRL production and serum PRL levels 15.

Actually other suggested mechanisms by the scientific community are: viral myocarditis (maybe the main mechanism [26], even if it is difficult to achieve a certain diagnosis because of the lack of histologic evidence in many cases); a post-partum abnormal immune response against fetal cells or antigens located in maternal myocardial tissue [27]; an exaggerated cardiac pump response to physiological increased cardiac output and to concomitant physiological decreased afterload; a systemic inflammatory peripartum state, demonstrated by increased blood levels of CK or TNFalfa, and the apoptosis-pathways related with consequent myocardial cells loss [28]; the prolonged use of tocolytic drugs, like beta-sympathomimetics taken for at least 4 weeks [29]; nutritional disorders and selenium or other micronutrients deficiencies [30].

In our case all there was no evidence of viral infection, no autoimmune antibody positive, no complement fraction blood levels over there normality ranges, no nutritional disorders, this is support the theory that prl has had a main rule in our patient so that there was a great responsiveness to the therapy choosen.

Even though the several therapeutic options available for PPCM, this disease still actually represents a potentially life-threatening condition: mortality could be up to $19 \%$ and the need for transplant is from $6 \%$ to $11 \%$. Among patients survived only in $23 \%$ - $41 \%$ left ventricle function goes back to normality and it takes several months; on the other hand often cardiac pump is marked compromise causing an irreversible worsening of quality of life in these young women. 
Concerning our patient it's interesting to focus both on the severity and rapidity of cardiac pump dysfunction development and at the same time on the early and considerable recovery of left ventricular function in PPCM.

Every evidence about PPCM considering the poor outcome associated is important for improve its management and for obtain best results, bringing patients back to a great quality of life.

\section{References}

1. Triebel J, Clapp C, de la Escalera GM, Bertsch T. Remarks on the Prolactin Hypothesis of Peripartum Cardiomyopathy. Front Endocrinol (Lausanne). 2017; 8: 77.

PubMed: https://pubmed.ncbi.nlm.nih.gov/28443067/

2. Ware JS, Li J, Mazaika E, Yasso CM, DeSouza T, Cappola TP et al. Shared Genetic Predisposition in Peripartum and Dilated Cardiomyopathies. N Engl J Med. 2016; 374: 233-241.

PubMed: https://pubmed.ncbi.nlm.nih.gov/26735901/

3. Ankita Kumara SS. Peripartum cardiomyopathy: a review of three case reports. Jp J. 2012.

4. Bouzerda A. Cardiomyopathie du péripartum: à propos d'une observation et revue de la littérature Peripartum cardiomyopathy: about a case and review of the literature. Pan Afr Med J. 2016; 25: 21 PubMed: https://www.ncbi.nlm.nih.gov/pmc/articles/PMC5268752/

5. Huang $Y$, Chen T, Zhang M, Yang X, Ding G, et al. Successful management of fatal peripartum cardiomyopathy in a young pregnant woman: A case report. Medicine (Baltimore). 2018; 97: e0408. PubMed: https://pubmed.ncbi.nlm.nih.gov/29642207/

6. Baris L. Peripartum cardiomyopathy: disease or syndrome? Heart. 2019.

7. Ziccardi MR, Siddique MS. Peripartum Cardiomyopathy. StatPearls Publishing. 2020

8. Demakis JG, Rahimtoola SH, Sutton GC, Meadows WR, Szanto PB et al. Natural course of peripartum cardiomyopathy. Circulation. 1971; 44:1053-61.

PubMed: https://pubmed.ncbi.nlm.nih.gov/4256828/

9. Pearson GO, Veille JC, Rahimtoola S, Hsia J, Oakley CM, et al. Peripartum cardiomyopathy: National Heart, Lung, and Blood Institute and Office of Rare Diseases (National Institutes of Health) workshop recommendations and review. JAMA. 2000; 283: 1183-1188. PubMed: https://pubmed.ncbi.nlm.nih.gov/10703781/

10. Michael $\mathrm{C}$, Honigberg MM. Peripartum cardiomyopathy. Tratto il giorno 2020. 2019. https://www.bmj.com/content/364/bmj.k5287.long

11. Homans DV. Peripartum cardiomyopathy. N Engl J Med. 1985; 312 1432-1427.

PubMed: https://pubmed.ncbi.nlm.nih.gov/3887170/

12. Isogai $T$, Kamiya $C A$. Worldwide Incidence of Peripartum Cardiomyopathy and Overall Maternal Mortality. Int Heart J. 2019; 60: 503-511.

PubMed: https://pubmed.ncbi.nlm.nih.gov/31019181/

13. Arany Z, Elkayam U. Peripartum Cardiomyopathy. Circulation. 2016; 133: 1397-409.

PubMed: https://pubmed.ncbi.nlm.nih.gov/27045128/

14. Breen TW, Janzen JA. Pulmonary hypertension and cardiomyopathy: anaesthetic management for Caesarean section. Can J Anaesthe. 1991; 38: 895-899.

PubMed: https://pubmed.ncbi.nlm.nih.gov/1742825/
15. Shaikh N, Ummunnisa F, Chanda A, Imran MA, Ganaw A, et al. Peripartum Cardiomyopathy: Facts and Figures. Tratto da. 2019. https://www.intechopen.com/books/inflammatory-heart-diseases/ peripartum-cardiomyopathy-facts-and-figures

16. Okeke TC, Ezenyeaku C, Ikeako L. Peripartum Cardiomyopathy. Ann Med Health Sci Res. 2013; 3; 313-319.

PubMed: https://pubmed.ncbi.nlm.nih.gov/24116305/

17. Guidelines EC. Cardiovascular Diseases during Pregnancy (Management of) Guidelines. 2018

18. Lata I, Gupta R, Sahu S, Singh H. Emergency management of decompensated peripartum cardiomyopathy. J Emerg Trauma ShocK. 2009; 2: 124-128.

PubMed: https://pubmed.ncbi.nlm.nih.gov/19561973/

19. Antila S, Sundberg S, Lehtonen LA. Clinical pharmacology of levosimendan. Clin Pharmacokinet. 2007; 46: 535-552. PubMed: https://pubmed.ncbi.nlm.nih.gov/17596101/

20. Benezet-Mazuecos J, de la Hera J. Peripartum cardiomyopathy: a new successful setting for levosimendan. Int J Cardiol. 2008; 123: 346-347. PubMed: https://pubmed.ncbi.nlm.nih.gov/17324479/

21. EstradaVHN,FrancoDLM,MorenoAAV, GambasicaJAR, BornacelliYEJ, et al. Reversion of Severe Mitral Insufficiency in Peripartum CardiomyopathyUsingLevosimendan.JClinMedRes.2015; 7:998-1001. PubMed: https://pubmed.ncbi.nlm.nih.gov/26566415/

22. Sieweke JT, Pfeffer TJ, Berliner D, König T, Hallbaum M, et al. Cardiogenic shock complicating peripartum cardiomyopathy: Importance of early left ventricular unloading and bromocriptine therapy. Eur Heart J Acute Cardiovasc Care. 2020; 9: 173-182. PubMed: https://pubmed.ncbi.nlm.nih.gov/29792513/

23. Mahmod M, Chan CF, Ali A, Raza S, Wan-Ibrahim NR, et al Cabergoline as an adjunct for early left ventricular recovery in peripartum cardiomyopathy. $\mathrm{Br} \mathrm{J}$ Cardiol. 2011; 18: 243-245.

24. Hilfiker-Kleiner D, Kaminski K, Podewski E, Bonda T, Schaefer A, Sliwa $\mathrm{K}$, et al. A Cathepsin D-Cleaved $16 \mathrm{kDa}$ Form of Prolactin Mediates Postpartum Cardiomyopathy. Cell. 2007; 128: 589-600. PubMed: https://pubmed.ncbi.nlm.nih.gov/17289576/

25. Bollen IA, V. D. Peripartum cardiomyopathy and dilated cardiomyopathy: different at heart. Front Physiol. 2015; 5 .

PubMed: https://pubmed.ncbi.nlm.nih.gov/25642195/

26. Mubarik A, Chippa V, Iqbal AM. Postpartum Cardiomyopathy. StatPearls Publishing. 2020.

PubMed: https://www.ncbi.nlm.nih.gov/books/NBK534770/

27. Shaikh N. An obstetric emergency called peripartum cardiomyopathy! J Emerg Trauma Shock. 2010; 3: 39-42.

PubMed: https://pubmed.ncbi.nlm.nih.gov/20165720/

28. Cowgill JA, Anthracycline SA, Sawyer DB. Peripartum Cardiomyopathies. Circ Res. 2019; 124: 1633-1646.

PubMed: https://pubmed.ncbi.nlm.nih.gov/31120822/

29. Lampert MB, Hibbard J, Weinert L, Briller J, Lindheimer M, et al Peripartum heart failure associated with prolonged tocolytic therapy. Am J Obstet Gynecol. 1993; 168: 493-495.

PubMed: https://pubmed.ncbi.nlm.nih.gov/8438916/

30. Fett JD, Ansari AA, Sundstrom JB, Combs GF. Peripartum cardiomyopathy: a selenium disconnection and an autoimmune connection. Int J Cardiol. 2002; 86: 311-316.

PubMed: https://pubmed.ncbi.nlm.nih.gov/12419571/ 